\title{
Physical Medicine and Rehabilitation Research
}

\section{Effect of high-heeled shoes on postural control in the upright and the leaning body stance}

\author{
Analina A Emmanouil and Elissavet N Rousanoglou* \\ Sport Biomechanics Lab, Sector of Sport Medicine \& Biology of Exercise, School of Physical Education \& Sport Science, National \& Kapodistrian University of \\ Athens, Greece
}

\begin{abstract}
Objectives: The studies examining the high-heeled shoes effect use the typical model of upright quiet stance with no information about leaning stances. This study aimed to examine the effect of high-heeled shoes in the upright and the leaning stance.

Methods: The trajectory of the center of pressure (COP) was recorded (forceplate Kistler-9286AA, sampling at 100 Hz, software Kistler BioWare -2812A1, v.3.2.6) in 11 young women $(22.14 \pm 2.93$ years, moderate users of high-heeled shoes) in the quiet upright and the leaning stance (forward, backward, rightward, leftward rigid body leaning). The participants were tested barefoot (BF) and with two high-heeled shoes (heel height- $\mathrm{HH}$ : $6.5 \mathrm{~cm}$ and $11 \mathrm{~cm}$ ). In the upright stance, the anterior-posterior and the medial-lateral COP path $(\mathrm{cm})$ and COP range $(\mathrm{cm})$, respectively, were determined. In the leaning stance, the COP displacement $(\mathrm{cm})$ and the COP range of stability ( $\%$ of BS length and $\%$ of BS width) were determined. In each stance, a repeated measures ANOVA followed by HH pairwise comparisons were used for statistics ( $\mathrm{p} \leq 0.05$, SPSS 22.0).

Main outcomes and results: In the upright stance, the COP path was significantly increased ( $\mathrm{p} \leq 0.05$ ) in the high-heeled shoes compared to BF, as well as when the $\mathrm{HH}$ was increased. The COP range was not significantly altered ( $\mathrm{p}>0.05)$. In the leaning stance, both the COP displacement and the COP range of stability were significantly altered $(\mathrm{p} \leq 0.05)$, with a directional sensitivity of the significant alterations in the COP range of stability.

Conclusions: The high-heeled shoes induce COP alterations which indicate a worsening of postural control in both the upright and the leaning stance, with a directional sensitivity in the leaning stance. Thus, the body positions that challenge postural control more than the typical upright stance should be also included in relevant studies.
\end{abstract}

Abbreviations: COP: Center of Pressure, HH: Heel height, BF: Barefoot, BS: Base of support, COM: Center of mass.

\section{Introduction}

The high-heeled shoes induce substantial noise in the process of postural control as they cause not only a reduction of the base of support (BS) but also shift upward the body center of mass (COM) $[1,2]$. Furthermore, the long-term wearing of high-heeled shoes is associated with muscle imbalance and instability around the ankle joint, a condition that increases the predisposition to an ankle sprain [3] and a fall incidence [4]. Thus, for an optimum body balance, the shoe heel height should range from 3 to 5 centimeters $[4,5]$.

The reduced postural control due to high-heeled shoes is evidenced in the significantly altered trajectory of the center of pressure (COP) [6-9]. In the relevant studies, the position of the upright quiet stance is typically used [6-9] with a lack of research information about leaning stances. In body leaning, the COM is shifted closer to the BS limits; thus, the postural control system operates at the borders of postural balance [10] where stability is achieved through a different strategy than the upright quiet standing [10-12]. In the quiet upright standing, the ankle (or the hip) strategy is used, whereas, the leaning stance brings the body at the border between the ankle and the stepping strategy [10-11]. In a rigid body leaning stance, the fast detection of the border between the ankle and the stepping strategy is crucial for postural stability [10-11]. The detection of the border between the ankle and the stepping strategy may become more difficult when wearing high-heeled shoes as the
COM is moved closer to the BS limits (closer to the postural stability limits). The leaning stance is an established biomechanical model for testing postural balance $[10,11,13]$, with some studies even questioning the appropriateness of using quiet stance as a general model of postural control $[13,14]$. Thus, this study aimed to examine the effect of highheeled shoes on postural control in the upright and the leaning body stance.

\section{Methods \\ Participants}

Eleven young women (Age: $22.14 \pm 2.93 \mathrm{yrs}$, Height: $1.63 \pm 0.04 \mathrm{~m}$, Weight: $52.44 \pm 6.94 \mathrm{~kg}$, BMI: $19.85 \pm 2.99 \mathrm{~kg} / \mathrm{m}^{2}$ ) participated in the study. The age of the young women was within the age range (20 to 29 years old) that the highest rate of injuries due to the use of high heel shoes is reported (34.2\% of cases from 2002 to 2012 [15], and $33.4 \%$ of cases from 2006 to 2010 [16]). Their anthropometric characteristics

${ }^{\star}$ Correspondence to: Elissavet Rousanoglou, Sport Biomechanics Lab, Sector of Sport Medicine \& Biology of Exercise, School of Physical Education \& Sport Science, National \& Kapodistrian University of Athens, Greece, E-mail: erousan@phed.uoa.gr

Key words: center of pressure, base of support, balance strategy, limits of stability

Received: September 14, 2018; Accepted: September 25, 2018; Published: September 28, 2018 
[17] and their high-heeled shoes wearing experience [7,8] are reported to affect postural control. Thus, with regard to body height, body mass and foot length, the inclusion criteria aimed to a body height range from $1.60 \mathrm{~m}$ to $1.70 \mathrm{~m}$, a normal BMI $\left(18.50 \mathrm{~kg} / \mathrm{m}^{2}-24.99 \mathrm{~kg} / \mathrm{m}^{2}\right)$ and participants who wore a 37 European shoe size $(6.5$ United States shoe size). With regard to the high-heeled shoes wearing experience, our participants wore shoes with heel height greater than $4 \mathrm{~cm}$ and less than $10 \mathrm{~cm}$ from 1 to 2 times a week, for 5-6 hours per day. Their wearing experience may be considered as moderate high-heeled shoes experience [7]. All participants had normal vision and hearing, and they were all free of any medical issue that would potentially influence postural stability. A written informed consent was obtained from all subjects. All experimental procedures were in accordance with the research policy of the School of Physical Education and Sports Science, National and Kapodistrian University of Athens, Greece.

\section{Procedures}

A force plate $(60 \mathrm{~cm} \times 40 \mathrm{~cm} \times 3,5 \mathrm{~cm}$, Kistler Type: 9286AA, Switzerland, sampling rate at $100 \mathrm{~Hz}$, BioWare $^{\oplus}$ data acquisition and analysis software-Kistler 2812A1, version 3.2.6.) was used to collect the COP trajectory data during two stance tests, the upright (quiet standing) and the leaning stance (Figure 1). Two trials were performed in each task with two minutes rest between trials.

Both the upright and the leaning task were performed barefoot (BF) and at two heel height $(\mathrm{HH})$ conditions: with a shoe of $6.5 \mathrm{~cm} \mathrm{HH}$, and with a shoe of $11 \mathrm{~cm} \mathrm{HH}$ (Figure 1). The order of each stance conditions was randomly assigned to the participants. To minimize any possible effect of the coefficient of friction [18] or footwear comfort [19] both shoes were similar in construction with the single exception of the $\mathrm{HH}$. According to the $\mathrm{HH}$ classification in previous studies (Table 1), the $6.5 \mathrm{~cm}$ and the $11 \mathrm{~cm} \mathrm{HH}$ of the present study may be classified as a medium and a high $\mathrm{HH}$, respectively. As it is presented in Table 1, the previous studies report as high-heeled shoes those with a $\mathrm{HH}$ from 9 to $10 \mathrm{~cm}$. Thus, the $11 \mathrm{~cm} \mathrm{HH}$ used in the present study could also be classified as a very high $\mathrm{HH}$.

In both the upright and the leaning stance, the participants were instructed to stand on the force plate, with feet comfortably spaced, their arms hanging at the side of their body. They were also asked to look at a circular target (diameter $3 \mathrm{~cm}$ ) positioned in front of them at eye level and at a distance of $3.5 \mathrm{~m}$. Sampling duration for upright stance was
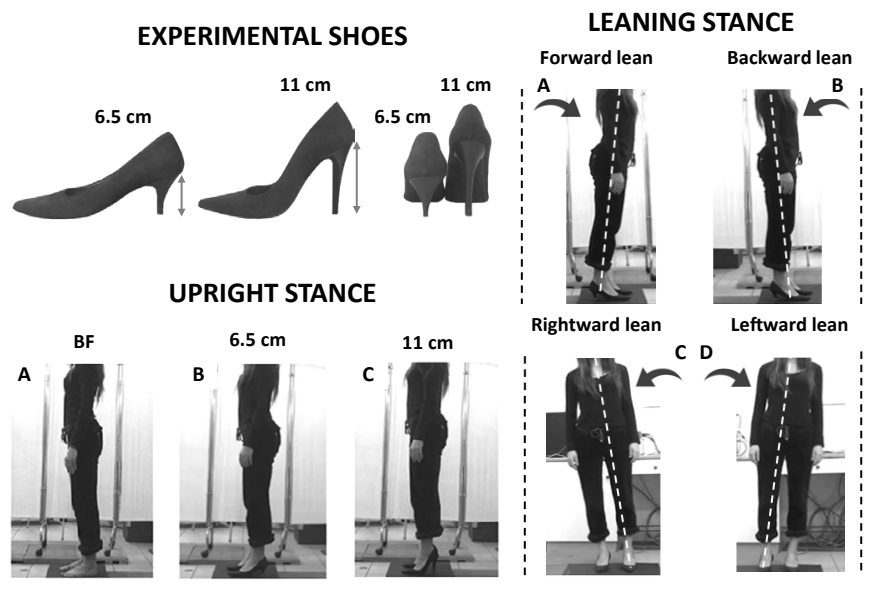

Figure 1. Left-Top: The two high-heeled experimental shoes (Medium HH: $6.5 \mathrm{~cm}$, High $\mathrm{HH}: 11 \mathrm{~cm})$. Left-Bottom: Upright stance in the barefoot (BF) and the high-heeled shoes (6.5 $\mathrm{cm} \mathrm{HH}$ and $11 \mathrm{~cm} \mathrm{HH})$ conditions. Left: The forward (A), the backward (B), the rightward (C) and the leftward (D) leaning stance
Table 1. Heel base width and heel height classification as used in previous studies with young women

\begin{tabular}{|c|c|c|c|c|c|c|c|}
\hline & $\begin{array}{c}\text { Age } \\
\text { (years) }\end{array}$ & $\begin{array}{c}\text { Heel base } \\
\text { width }\end{array}$ & & \multicolumn{4}{|c|}{ Heel height (cm) $^{\dagger}$} \\
\cline { 5 - 9 } & BF & Flat & Low & Medium & High \\
\hline $\begin{array}{c}\text { Gerber, et al. } \\
\text { (2012) }\end{array}$ & $18-30$ & $1 \mathrm{~cm} \times 1 \mathrm{~cm}$ & BF & & & & 7 \\
\hline Ko, et al. (2013) & $20.9 \pm 1.3$ & not reported & & 0.5 & 4 & & 9 \\
\hline Kim, et al. (2013) & $22.9 \pm 1.5$ & not reported & & 0 & & & 7 \\
\hline $\begin{array}{c}\text { Hapsari and } \\
\text { Xiong (2015) }\end{array}$ & $18-30$ & $1.5 \mathrm{~cm} \times 1.5 \mathrm{~cm}$ & & 1 & 4 & 7 & 10 \\
\hline Present study & $22.1 \pm 2.9$ & $1 \mathrm{~cm} \times 1 \mathrm{~cm}$ & $\mathrm{BF}$ & & & 6.5 & 11 \\
\hline
\end{tabular}

$\dagger$ the heel height classification as reported in each study. ${ }^{\dagger \dagger} \mathrm{BF}$ : barefoot

chosen at $70 \mathrm{~s}$ [6-9]. In the leaning stance, the same instructions were provided while, in addition, the participants were further instructed to subsequently perform a forward, a backward, a rightward and a leftward maximum body lean, and to maintain each lean for $5 \mathrm{~s}$ [20]. Each body lean was performed as far as possible while maintaining a straight body line from heel to head, and both heels in contact with the force plate. Between each body lean directional change, the participants returned to the upright quiet position where they remained for $5 \mathrm{~s}$. Based on pilot data collection, the total sampling duration for the leaning stance was $80 \mathrm{~s}$.

The perimeter of the feet in the BF condition, as well as the perimeter defined by the sole and the heel of the shoe in the two $\mathrm{HH}$ conditions, was traced on a piece of paper placed on the top of the force plate. Thus, in every trial, each participant repositioned herself in the same place with regard to the force platform coordinates. The traced perimeter was used to define the dimensions of the base of support (BS) (Figure 2). The correct repositioning of each participant on the BS trace was carefully checked. The BS was measured at $27.73 \pm 2.07 \mathrm{~cm}, 27.71 \pm$ $2.07 \mathrm{~cm}$ and $27.71 \pm 2.07 \mathrm{~cm}$, for $\mathrm{BF}$, for the $6.5 \mathrm{~cm} \mathrm{HH}$ and the $11 \mathrm{~cm}$ $\mathrm{HH}$, respectively. The BS width was significantly larger (paired t-test, $\mathrm{p} \leq 0.05)$ in $\mathrm{BF}$ than the $6.5 \mathrm{~cm} \mathrm{HH}(\mathrm{p}=0.040)$ and $11 \mathrm{~cm} \mathrm{HH}(\mathrm{p}=$ 0.040 ) shoes, with no significant difference between the two shoes ( $p$ $>0.05$ ). In the BF condition, the BS length was measured at $23.71 \pm$ $0.79 \mathrm{~cm}$, while in the shoe condition the BS length was fixed at 22.5 $\mathrm{cm}$ and at $21 \mathrm{~cm}$, for the $6.5 \mathrm{~cm} \mathrm{HH}$ and the $11 \mathrm{~cm} \mathrm{HH}$, respectively. In the $\mathrm{BF}$ condition, the feet opening angle was calculated according to Chiari et al. [17] and was found at $11.28 \pm 3.23$ degrees, based on the distance between the two big toes $(14.55 \pm 3.36 \mathrm{~cm})$ and the intermalleolar distance $(9.86 \pm 2.89 \mathrm{~cm})$. The variability $(26.8 \%)$ of the feet opening angle is within the BS stance expected range which may be considered as a low foot opening angle variability [21]. To the best of our knowledge, no calculation method has been previously reported for the feet opening angle in a shoe wearing stance.

Data processing: In the upright stance, the variables determined were the COP path $(\mathrm{cm})$ and the COP range $(\mathrm{cm})$, in the anteriorposterior and the medial-lateral direction, respectively. In the leaning stance, the variables determined were the COP displacement expressed in $\mathrm{cm}$, and the COP range of stability expressed as a percentage (\%) of the BS dimensions. The forward, the backward, the rightward, and the leftward COP displacement $(\mathrm{cm})$ was defined as the difference between the mean COP value during the $5 \mathrm{sec}$ that the participant remained at the upright stance and the mean COP value during the 5 sec that the participant remained at each direction, respectively, of the leaned body position. For the calculation of the COP range of stability calculation, initially, the total anterior-posterior and the total mediallateral COP displacements were estimated as follows: A) the sum of the maximum forward and the maximum backward COP displacement defined the total anterior-posterior displacement (displacement in the 


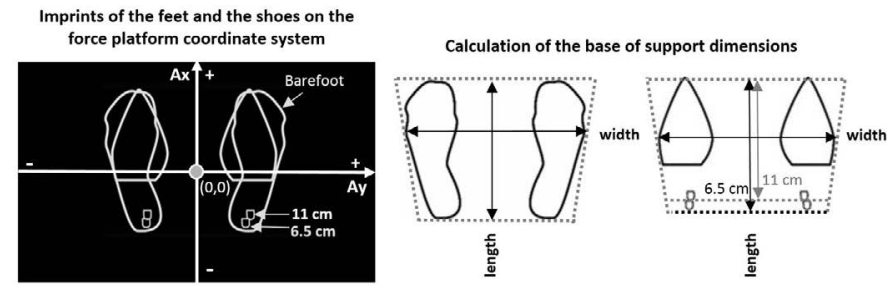

Figure 2. Left: Imprints of the feet and the shoes (heel height of $6.5 \mathrm{~cm}$ and $11 \mathrm{~cm}$ ) with regard to the force platform coordinate system (Ax: anterior-posterior axis, Ay: mediallateral axis, 0.0 : origin point). Right: Schematic representation of the base of support and of the calculation of its length and width dimension

direction of BS length) and B) the sum of the maximum rightward and the maximum leftward COP displacement defined the total mediallateral displacement (displacement in the direction of BS width). In continuance, two variables were defined for the COP range of stability, naming the anterior-posterior and the medial-lateral COP range of stability, expressed as a percentage (\%) of the BS length and the BS width, respectively. In both the upright and the leaning stance, the value that inserted in the statistical analysis was the two trial average for each stance condition.

\section{Statistical analysis}

A repeated measures ANOVA were conducted separately for the upright and the leaning stance, with 3 levels of the repeated factor in each one (BF, $6.5 \mathrm{~cm} \mathrm{HH}$ and $11 \mathrm{~cm} \mathrm{HH}$ ) to test the $\mathrm{HH}$ effect on the postural control variables. If the Mauchly's test of sphericity indicated a sphericity violation, the Greenhouse-Geisser $(\varepsilon<0.75)$ or the HuynhFeldt $(\varepsilon>0.75)$ corrections were used. If the $\mathrm{HH}$ main effect was significant, the pairwise comparisons with Bonferroni correction were tested between stance conditions. For the statistical tests, the level of significance was set at $\mathrm{p} \leq 0.05$. Statistical analysis was carried out using IBM SPSS Statistics (Version 22.0).

\section{Results}

Figure 3 illustrates an example of the COP path in each $\mathrm{HH}$ condition with regard to the feet positioning on the force plate for a representative subject in the upright and the leaning body stance.

Upright stance: In the upright stance, a significant $\mathrm{HH}$ effect was found for the COP path in both the anterior-posterior $(\mathrm{F}=46.38, \mathrm{p}=$ $0.000)$ and the medial-lateral $(\mathrm{F}=11.22, \mathrm{p}=0.000)$ direction (Figure 4). The $\mathrm{HH}$ differences with regard to the COP path were all significant, in both the anteroposterior and mediolateral direction $(\mathrm{p}=0.000$ for all), except for the comparison between $\mathrm{BF}$ and the $6.5 \mathrm{~cm}$ HH in the medial-lateral direction (Figure 4). The $\mathrm{HH}$ effect was not significant for the COP range in both the anterior-posterior $(\mathrm{F}=2.53, \mathrm{p}=0.105)$ and the medial-lateral $(\mathrm{F}=1.02, \mathrm{p}=0.379)$ direction (Figure 4).

Leaning stance: In the leaning stance, a significant $\mathrm{HH}$ effect was found for the COP displacement during the forward $(\mathrm{F}=94.42, \mathrm{p}=$ $0.000)$, the backward $(\mathrm{F}=33.75, \mathrm{p}=0.000)$, the rightward $(\mathrm{F}=8.96, \mathrm{p}$ $=0.000)$ and the leftward $(\mathrm{F}=25.97, \mathrm{p}=0.000)$ body lean (Figure 5). The HH differences in the COP displacement were all significant in the forward and the backward lean ( $\mathrm{p}=0.000$ for all). In the rightward lean, the difference was significant only between the $\mathrm{BF}$ and the $11 \mathrm{~cm} \mathrm{HH}(\mathrm{p}$ $=0.010)(p=0.089$ for BF versus $6.5, p=0.246$ for $6.5 \mathrm{~cm} \mathrm{HH}$ versus 11 $\mathrm{cm} \mathrm{HH}$ ) (Figure 5). In the leftward lean, the difference was significant only between the BF and the $6.5 \mathrm{~cm} \mathrm{HH}(\mathrm{p}=0.000)$, as well as between the $\mathrm{BF}$ and $11 \mathrm{~cm} \mathrm{HH}(\mathrm{p}=0.000)$, but not between the $6.5 \mathrm{~cm}$ and the $11 \mathrm{~cm} \mathrm{HH}(\mathrm{p}=1.000)$.
With regard to the COP range of stability, the $\mathrm{HH}$ effect was significant for both $B S$ dimensions (BS length: $F=102.65, p=0.00$, BS width: $\mathrm{F}=16.35, \mathrm{p}=0.00$ ) (Figure 6). The $\mathrm{HH}$ differences in the COP range of stability were all significant $(\mathrm{p}=0.000$ for all), except for the comparison between the $6.5 \mathrm{~cm}$ and the $11 \mathrm{~cm} \mathrm{HH}(\mathrm{p}=1.000)$ with regard to the BS width $(\mathrm{p}=1.000)$ (Figure 6).

\section{Discussion}

The purpose of this study was to examine the effect of high-heeled shoes on postural control in the upright and the leaning body stance. The assumption of the study was that, in both the upright stance and the leaning body position, the high-heeled shoes would cause a worsening of body balance, which would be more pronounced at increased $\mathrm{HH}$.

In the present study, the postural control worsening was documented at the increase of the COP path in the upright stance and, the reduction of the COP displacement and of the COP range of stability in the leaning stance. The worsened postural control found in the present study is in agreement with previous studies [1,6-9]. When

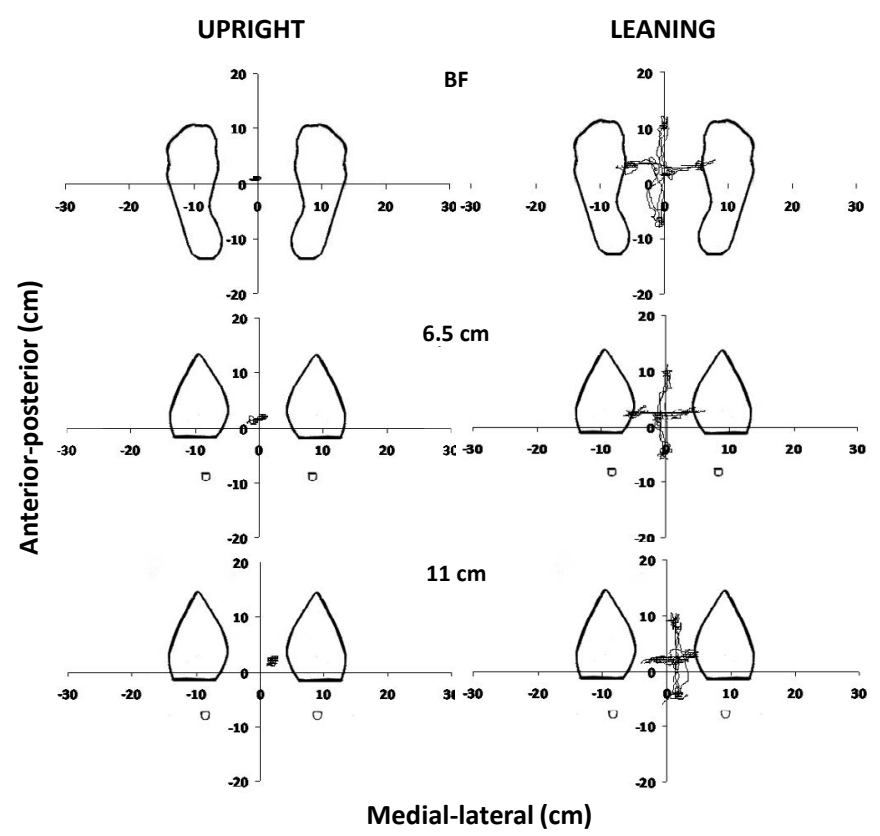

Figure 3. Schematic representation of the COP path of one subject with regard to the base of support, in the barefoot (BF) and the high-heeled shoes $(6.5 \mathrm{~cm} \mathrm{HH}$ and $11 \mathrm{~cm} \mathrm{HH})$ conditions, in the upright (Left) and the leaning (Right) stance. The anterior-posterior and the medial-lateral directions indicate the Ax and Ay, respectively axis of the force plate coordinate system
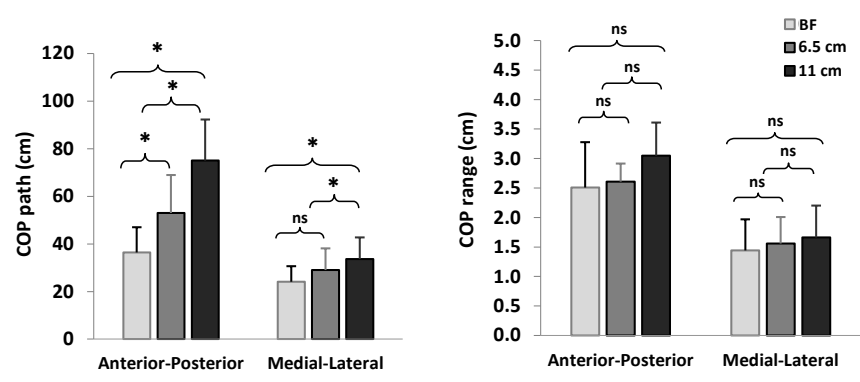

Figure 4. Mean (SD) of the COP path (Left) and the COP range (Right) in the uprigh stance, in the anterior-posterior and the medial-lateral directions, in the barefoot $(\mathrm{BF})$ and the high-heeled shoes $(6.5 \mathrm{~cm} \mathrm{HH}$ and $11 \mathrm{~cm} \mathrm{HH})$ conditions.*significant pairwise difference between HH conditions at $\mathrm{p} \leq 0.05$ 


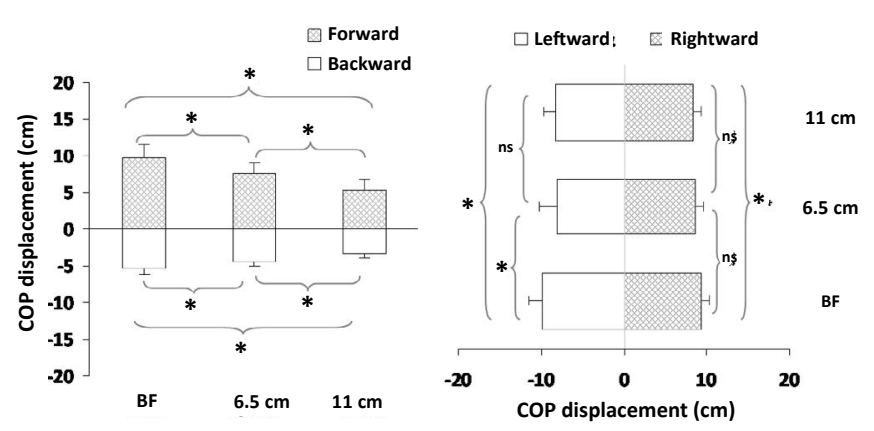

Figure 5. Mean (SD) of the leaning stance COP displacement, in the forward and backward lean (Left), as well as in the rightward and leftward lean (Right), in the barefoot (BF) and the high-heeled shoes $(6.5 \mathrm{~cm} \mathrm{HH}$ and $11 \mathrm{~cm} \mathrm{HH})$ conditions. *significant pairwise difference between $\mathrm{HH}$ conditions at $\mathrm{p} \leq 0.05$
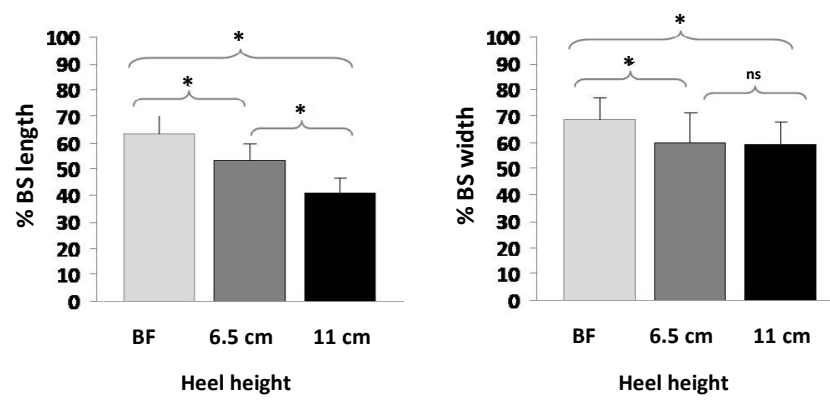

Figure 6. Mean (SD) of the leaning stance COP range of stability, expressed as a percentage (\%) of the base of support (BS) length (Left) and BS width (Right), in the barefoot (BF) and the high-heeled shoes $(6.5 \mathrm{~cm} \mathrm{HH}$ and $11 \mathrm{~cm} \mathrm{HH})$ conditions. *significant pairwise difference between $\mathrm{HH}$ conditions at $\mathrm{p} \leq 0.05$

wearing high-heeled shoes, the upward shift of COM [4] and the concomitant forward COP displacement [1,2] lead to changes of the foot joint configuration and in the muscle function around the ankle joint, that is the joint most proximal to the BS $[2,22]$. The change of the foot anatomical configuration towards an increased plantar flexion due to high-heeled shoes reduces the effectiveness of the ankle plantar flexors [22], thus hindering the optimal postural stability. The increased plantar flexion due to high-heeled shoes also induces modifications of the normal anatomical configuration [4] and the normal muscle function $[4,22]$ in body areas distal to the BS. These distal modifications further hinder the postural control when wearing high-heeled shoes, and are associated with musculoskeletal pain $[4,22]$.

In the relevant studies, the $\mathrm{HH}$ from 9 to $10 \mathrm{~cm}$ is typically classified a high heel condition [6-9]; nevertheless, there appear no distinct criteria of the $\mathrm{HH}$ classification as flat, low, medium or high. Furthermore, the width of the heel width base is not always reported [6-9], although it is considered as a critical parameter for postural stability [23]. In specific, the foot is more unstable with a small than with a large heel base as shown by the COP trajectories and the plantar pressure distribution in walking [23]. For an optimal foot functionality and the avoidance of harmful fatigue, the shoe $\mathrm{HH}$ should range from 3 to $5 \mathrm{~cm}$ (usually classified as low $\mathrm{HH}$ ) [4,6-9]. However, in the present study, the $11 \mathrm{~cm}$ $\mathrm{HH}$ was chosen as it is very popular among young women; in addition, its small heel base width $(1 \mathrm{~cm} \mathrm{x} 1 \mathrm{~cm})$ indicates a very restricted BS. Thus, it was expected to impose a substantial challenge to postural control, particularly in the leaning body stance which inherently brings the COM closer the BS borders.

In the leaning stance, the significant reduction of the forward and backward COP displacement when $\mathrm{HH}$ was increased is in agreement with previous studies [7]. The differences between the BF and the highheeled conditions, as well as between the two $\mathrm{HH}$, were all significant in both the forward and backward lean; however, this was not the case in the rightward and the leftward lean. This finding may be associated with the asymmetry of body weight distribution, as normal adults appear to load more their right foot for about $60 \%$ of their stance time [24], but it may also indicate a directional sensitivity of postural stability $[25,26]$. The directional sensitivity of postural control as a function of the BS geometry has been previously documented in dynamic and static conditions [26]. The postural stability during the upright tandem stance showed a 3-fold greater sensitivity in the medial-lateral direction (BS width) than the anterior-posterior direction (BS length) [26]. The reverse was observed in the typical upright stance, where the postural stability may be 2.3 -fold greater in the anterior-posterior direction (BS length) than the medial-lateral direction (BS width) [26]. It is possible that, besides BS geometry, the directional sensitivity of postural control may also depend on the inherent closeness of the COM projection to the BS borders in the stance under examination (as in the case of highheeled leaning), a situation that may alter the proprioceptive control of posture [25].

In the $\mathrm{HH}$ of $11 \mathrm{~cm}$ which is particularly popular among young women, the significant decrease of the COP stability range (64\%, 56\% and $41 \%$ of the BS length in the BF, the $6.5 \mathrm{~cm} \mathrm{HH}$ and the $11 \mathrm{~cm} \mathrm{HH}$, respectively) underlines the reduction of the utilized BS length when wearing high-heeled shoes. The reduction of the utilized BS length when wearing high-heeled shoes may be associated with the proprioceptive detection of the transition border between the ankle and the stepping postural control strategy [10-12]. These two strategies involve different muscle synergies; thus, for a successful postural control, the fast detection of the transition border from one strategy to the other is of critical importance $[10,11]$. When compared to the upright stance, the leaning stance imposes an inherent noise to the postural control system due to the COM projection as closer as possible to the BS limits, or more accurately, as closer as possible to the border between the ankle and the stepping strategy [10-13]. In high-heeled compared to BF leaning, this inherent noise may be further accentuated, allowing the assumption of an enhanced difficulty to encode the border between the ankle and the stepping strategy. Besides the reduction of the utilized BS length, there was also a significant reduction of the utilized BS width in the high-heeled leaning compared to BF leaning, but not when the shoe $\mathrm{HH}$ was increased $(71 \%, 61 \%$ and $60 \%$ of the BS width in the BF, the $6.5 \mathrm{~cm} \mathrm{HH}$ and the $11 \mathrm{~cm} \mathrm{HH}$, respectively). In specific, the reduction in the utilized BS width (about 10 percentage points) appeared lower than the reduction in the utilized BS length ( 8 and 23 percentage points, respectively in the $6.5 \mathrm{~cm}$ and the $11 \mathrm{~cm} \mathrm{HH}$ compared to $\mathrm{BF}$, and 15 percentage points between the two $\mathrm{HH}$ ). Overall, the utilized BS reductions may indicate a directional sensitivity of postural control, a concept that has been previously documented [26] and has been associated with situations that may alter the proprioceptive control of posture [25].

The comparison of the leaning stance results with previous studies is not easy as, to the best of our knowledge, no previous study appears to examine the $\mathrm{HH}$ effect in a rigid body leaning stance as the one used in the present study. Hapsari and Xiong [7] who used a task including body leaning in their protocol, also report that the $\mathrm{HH}$ increase $(0$ $\mathrm{cm}, 4 \mathrm{~cm}, 7 \mathrm{~cm}$, and $10 \mathrm{~cm}$ ) resulted to a significant postural control worsening, particularly when $\mathrm{HH}$ was increased more than $4 \mathrm{~cm}$. However, the task used in the protocol of Hapsari and Xiong [7] was a complex task in which postural control (biomechanical stability) was a secondary function. In the task of Hapsari and Xiong [7], the 
performance stability rather than the biomechanical stability was the primary function; furthermore, the leaning position was not a rigid body position, as their task could be performed with or without hip flexion. Both the upright quiet stance $[11,12]$ and the rigid body leaning stance [10-13] are established models for testing the biomechanical stability of the postural control. The upright quiet stance is predominantly used in postural control studies; however, it should be noted that some studies question the appropriateness of the upright quiet as a general model of postural control $[13,14]$.

\section{Conclusion}

In the present study, in both the upright and leaning stance, the COP alterations when wearing high-heeled shoes denote a postural control worsening which may be associated to a decreased postural stability and an increased falling risk. The postural control was further worsened when the $\mathrm{HH}$ was increased, with a directional sensitivity of the postural control in the leaning stance. One could claim that the lessening of wearing high-heeled shoes, or even the abolition of highheeled shoes, could lead to an effective elimination of all the negative issues that arise from their use. However, despite the public warnings from institutions of occupational health [27] and international medical societies [28], it is possible that, for a presentable image or a formal office wear, employers may enforce to women a dress code that includes the daily long-hour use of high-heeled shoes [27-29]. The increasing prevalence of daily high-heeled shoes wearing is ascertained (from $39 \%$ up to $49 \%$ of the women population in the period for from 2003 to 2014) [28]. The 2-fold increase in the injury prevalence due to high-heeled shoes is also ascertained for the period from 2002 to 2012 [15,16]. Thus, the studies that highlight the acute or overuse injury risk potential due to high-heeled shoes are of critical importance. Such studies should include not just the typical model of upright quiet stance, but also body positions that challenge postural control and may provide indisputable evidence of the potential harmful implications of high-heeled shoes wearing.

\section{Competing interest declaration}

The authors declare that they have no competing interest.

\section{References}

1. Cronin NJ (2014) The effects of high heeled shoes on female gait: a review. $J$ Electromyogr Kinesiol 24: 258-263. [Crossref]

2. Bae YH, Ko M, Park YS, Lee SM (2015) Effect of revised high-heeled shoes on foot pressure and static balance during standing. J Phys Ther Sci 27: 1129-1131. [Crossref]

3. Gefen A, Megido-Ravid M, Itzchak Y, Arcan M (2002) Analysis of muscular fatigue and foot stability during high-heeled gait. Gait Posture 15: 56-63. [Crossref]

4. Lee M, Jeong E, Freivalds A (2001) Biomechanical effects of wearing high-heeled shoes. Int J Ind Ergon 28: 321-326.

5. Lee KY, Lee HJ, Kim SE, Choi PB, Song SH, et al. (2012) Short term rehabilitation and ankle instability. Int J Sports Med 33: 485-496. [Crossref]

6. Gerber S, Costa R, Grecco L, Pasini H, Marconi N, et al. (2012) Interference of highheeled shoes in static balance among young women. Hum Mov Sci 31: 1247-1252. [Crossref]
7. Hapsari V, Xiong S (2015) Effects of high heeled shoes wearing experience and heel height on human standing balance and functional mobility. Ergonomics 59: 249-264. [Crossref]

8. Kim Y, Lim JM, Yoon B (2013) Changes in ankle range of motion and muscle strength in habitual wearers of high-heeled shoes. Foot Ankle Int 34: 414-419. [Crossref]

9. Ko DY, Lee HS (2013) The changes of cop and foot pressure after one hour's walking wearing high-heeled and flat shoes. J Phys Ther Sci 25: 1309-1312. [Crossref]

10. Blaszczyk JW, Hansen PD, Lowe DL (1993) Postural sway and perception of the upright stance stability borders. Perception 22: 1333-1341. [Crossref]

11. Horak FB, Moore SP (1993) The effect of prior leaning on human postural responses. Gait Posture 1: 203-210.

12. Winter DA (1995) Human balance and posture control during standing and walking. Gait Posture 3: 193-214.

13. Riley MA, Mitra S, Stoffregen TA, Turvey MT (1997) Influences of body lean and vision on unperturbed postural sway. Motor Control 1: 229-246.

14. Stoffregen TA, Adolph K, Thelen E, Gorday KM, Sheng YY (1997) Toddlers postural adaptations to different support surfaces. Motor Control 1: 119-137.

15. Moore JX, Lambert B, JenkinS GP, Mcgwin G Jr (2015) Epidemiology of high-hee shoe injuries in U.S. women: 2002 to 2012. J Foot Ankle Surg 54: 615-619. [Crossref]

16. Williams CM, Haines TP (2014) An exploration of emergency department presentations related to high heel footwear in Victoria, Australia, 2006-2010. J Foot Ankle Res 7: 4. [Crossref]

17. Chiari L, Rocchi L, Capello A (2002) Stabilometric parameters are affected by anthropometry and foot placement. Clin Biomech (Bristol, Avon) 17: 666-677. [Crossref]

18. Blanchette MG, Brault JR, Powers CM (2011) The influence of heel height on utilized coefficient of friction during walking. Gait Posture 34: 107-110. [Crossref]

19. Witana CP, Goonetilleke RS, Au EY, Xiong S, Lu X (2009) Footbed shapes for enhanced footwear comfort. Ergonomics 52: 617-628. [Crossref]

20. Frames E, Soangra R, Lockhar TE (2013) Assessment of postural stability using inertial measurement unit on inclined surfaces in healthy adults. Biomed Sci Instrum 49: 234 242. [Crossref]

21. Mcllroy WE, Maki BE (1997) Preferred placement of the feet during quiet stance: development of a standardized foot placement for balance testing. Clin Biomech (Bristol, Avon) 12: 66-70. [Crossref]

22. Esenyel M, Walsh K, Walden J, Gitter A (2003) Kinetics of high-heeled gait. $J A m$ Podiatr Med Assoc 93: 27-32. [Crossref]

23. Luximon Y, Cong Y, Luximon A, Zhang M (2015) Effects of heel base size, walking speed, and slope angle on center of pressure trajectory and plantar pressure when wearing high-heeled shoes. Hum Mov Sci 41: 307-319. [Crossref]

24. Gutnik B, Leaver J, Standen C, Longley C (2008) Inferred influence of human lateral profile on limb load asymmetry during a quiet standing balance test. Acta Med Okayama 62: 175-184. [Crossref]

25. Allum JH, Bloem BR, Carpenter MG, Hulliger M, Hadders-Algra M (1998) Proprioceptive control of posture: a review of new concepts. Gait Posture 8: 214-242. [Crossref]

26. O'Connor SM, Kuo AD (2009) Direction-dependent control of balance during walking and standing. J Neurophysiol 102: 1411-1419. [Crossref]

27. Trades Union Congress-TUC (2011) Working feet and footwear: Health and safety at work guidance, ppa.

28. American Podiatric Medical Association (2014) public opinion research on foot health and care findings from a survey of 1000 U.S. adults.

29. Chua YP, Tan WJ, Yahya TS, Saw A (2013) Prevalence of nontraumatic foot pain among urban young working women and its contributing factors. Singapore Med J 54: 630-633. [Crossref]

Copyright: (C2018 Emmanouil AA. This is an open-access article distributed under the terms of the Creative Commons Attribution License, which permits unrestricted use, distribution, and reproduction in any medium, provided the original author and source are credited. 